\title{
Systemic melatonin application increases bone formation in mandibular distraction osteogenesis
}

\begin{abstract}
Izzet ACIKAN(a)
Mehmet GUL(b)

Gokhan ARTAS(c)

Ferhan YAMAN(d)

Gulnihal DENIZ

Ozgur BULMUS ${ }^{(f)}$

Mustafa KOM(g)

Mustafa KIRTAY(h)

Serkan DUNDAR ${ }^{(i)}$
\end{abstract}

(a)Sutcu Imam University, Faculty of Dentistry, Department of Oral and Maxillofacial

Surgery, Kahramanmaras, Turkey.

(b) Harran University, Faculty of Dentistry, Department of Periodontology, Sanliurfa, Turkey.

(c) Firat University, Faculty of Medicine, Department of Medical Pathology, Elazig, Turkey.

(d) Private Practice, Oral and Maxillofacial

Surgeon, Diyarbakır, Turkey.

(e) Firat University, Faculty of Medicine,

Department of Anatomy, Elazig, Turkey.

(f) Firat University, Faculty of Medicine, Department of Medical Physiology, Elazig, Turkey.

(g) Firat University, Faculty of Veterinary Medicine, Department of Surgery, Elazig, Turkey.

(h) Private Practice, Oral and Maxillofacial

Surgeon,Toronto, Canada.

(i)Firat University, Faculty of Dentistry, Department of Periodontology, Elazig, Turkey

Declaration of Interests: The authors certify that they have no commercial or associative interest that represents a conflict of interest in connection with the manuscript.

\section{Corresponding Author:}

Izzet Acikan

E-mail: dtizzetacikan@hotmail.com

https://doi.org/10.1590/1807-3107bor-2018.vol32.0085

Submitted: February 11, 2018

Accepted for publication: June 27, 2018

Last revision: July 16, 2018
Abstract: This study aimed to investigate the effects of different doses of systemic melatonin application on new bone formation during mandibular distraction osteogenesis (DO) in rats. Mandibular DO was performed on 30 adult female Sprague-Dawley rats, which were randomly divided into three groups: control group (CNT), melatonin dose 1 (MLT-D1), and melatonin dose 2 (MLT-D2). A five-day latent waiting period and a ten-day distraction phase followed the surgery. After the surgery, rats from the MLT-D1 and MLT-D2 groups received 25 and $50 \mathrm{mg} / \mathrm{kg}$ melatonin, respectively, at 7, 14, 21, 28, and 35 days. The animals were euthanised 28 days after distraction, i.e. at 43 days after surgery. Histological and histomorphometric analyses revealed that the distracted bone area was completely filled with new bone formation in all three groups. The MLT-D2 group exhibited the most new bone formation, followed by MLT-D1 and CNT. The melatonin groups had more osteoclasts than the CNT $(\mathrm{p}<0.05)$. The number of osteoblasts was higher in the melatonin groups than in the CNT group, and the MLT-D2 had more osteoclasts than the MLT-D1 group $(\mathrm{p}<0.05)$. Finally, the osteopontin $(\mathrm{OPN})$ and vascular endothelial growth factor (VEGF) levels were higher in the melatonin groups than in the CNT group, and the MLT-D2 had higher OPN and VEGF levels than the MLT-D1 ( $\mathrm{p}<0.05)$. This study suggests that systemic melatonin application could increase new bone formation in DO.

Keywords: Melatonin; Pineal Gland; Osteogenesis, Distraction; Bone and Bones.

\section{Introduction}

Distraction osteogenesis (DO) is a reasonable treatment option for the reconstruction of maxillar and mandibular bone deficiencies and craniofacial anomalies, as it prevents the onset of donor site morbidity while generating both hard (jaw bone) and soft tissues. ${ }^{1,2}$ During the DO procedure, transduction of bone osteotomies using mechanical force at the bone fracture site stimulates a potent osteogenic and vasculogenic response in newly regenerated bone tissues, resulting in anisotropically oriented bone. $^{3}$ Although DO is a reliable method for treating jaw bone and soft tissue abnormalities, there are some limitations, particularly connected to long-term consolidation periods and the stability of regenerated bone. 
For these reasons, numerous techniques, methods, and materials have been researched to accelerate the maturation of regenerated bone, including hormones, calcium sulphate, growth factors, and electronic and ultrasonic stimulation. ${ }^{3,4}$

Osteoblastic cells, which are bone formative, and osteoclastic cells, which are bone resorptive, are both vital to DO. Together, the formation and resorption of bone tissue are known as bone remodelling. This process is actualized when osteoblasts and osteoclasts work together physiologically. ${ }^{1,23,4,5}$ In practice, the bone tissue remodelling mechanism is organized by the activity of systemic hormones (melatonin, oestradiol, parathyroid, and growth hormones), as well as by bone marrow and osteoid matrix-derived growth factors. Of particular interest among these systemic hormones is melatonin, because of its osteoblastic activity. ${ }^{5,6,7}$ Melatonin is a tryptophanderived indolamine hormone that is released by the pineal gland, but it is also found in many tissues such as the retina and intestinal mucosa. Research has shown that melatonin has well-known antioxidant capabilities and free radical scavenging potency. ${ }^{5,6} \mathrm{It}$ can also inhibit bone tissue resorption as it suppresses osteoclastogenesis. ${ }^{5,6,7}$ In addition to this, in an in vitro study, authors reported that melatonin can increase osteoblast proliferation and differentiation. 5,6,7 Thus, we proposed in the current work that the systemic application of melatonin during DO procedures would be an effective treatment technique for increased new bone formation. Thus, in this study we aimed to evaluate the histomorphometric effects of two different systemic melatonin applications on new bone regeneration during DO procedures in rat mandibles.

\section{Methodology}

\section{Animals}

To this end, we used 30 female 1- to 1.5-yearold Sprague-Dawley rats in the same oestrus cycle (because of the hormonal effects known in wound and bone healing). Their average body weights at the start of the study were $280-300 \mathrm{~g}$. The animals were kept in temperature-controlled cages (approximately $25^{\circ} \mathrm{C}$ ), exposed to a 12:12-hour light-dark cycle, and had access to food and water ad libitum. The study protocol and experimental design were approved by the Animal Experimental Ethics Committee, Dicle University.

\section{Experimental design}

The rats were divided into three groups as outlined below:

a. Control $(\mathrm{CNT})$ group $(\mathrm{n}=10)$ : No treatment was applied, and only DO was performed on the rat mandibles;

b. Melatonin dose 1 (MLT-D1) group ( $\mathrm{n}=10$ ): The rats received intraperitoneal injections of $25 \mathrm{mg} / \mathrm{kg}$ melatonin at 7, 14, 21, 28, and 35 days after surgery;

c. Melatonin dose 2 (MLT-D2) group ( $\mathrm{n}=10$ ): The rats received intraperitoneal injections of $50 \mathrm{mg} / \mathrm{kg}$ melatonin at 7, 14, 21, 28, and 35 days after surgery.

Melatonin was dissolved in ethanol and further diluted in physiological serum. The final concentration of ethanol was $1 \%$.

\section{Device design}

In order to construct the distractor used in applying DO, an orthodontic jackscrew embedded in acrylic resin was used, as reported previously. ${ }^{9}$ The distractor was stabilized with three titanium bone screws. The activation of each distractor facilitated a progressive distraction, because each activation of the distractor at $180^{\circ}$ resulted in $0.175 \mathrm{~mm}$ of separation in the osteotomized bone fragments. ${ }^{9}$

\section{Surgical procedure}

During the surgical procedures, general anaesthesia was administered using intramuscular injections of $35 \mathrm{mg} / \mathrm{kg}$ of ketamine hydrochloride and $5 \mathrm{mg} / \mathrm{kg}$ of xylazine, and the surgical operations were performed under sterile conditions. After the rats were anaesthetised, the mandibular skin was washed with povidone iodine, and the surgical area was shaved before the start of the surgery. A 2-cm submandibular skin incision was made while avoiding nerve and vessel damage. Next, the subcutaneous tissues were carefully exposed. A periosteal elevator was used to lift the flap and periosteum to reach the 
mandibular bone. The mandibular skin was then sutured with $4 / 0$ polyglactin absorbable sutures. All animals received intramuscular injections of an antibiotic, cephalosporin, and an analgesic, tramadol hydrochloride, for three days after the operation. The protocol for the surgical procedure has been reported previously. ${ }^{9,10}$ To ensure their standardization, the distraction devices were fixed to the right of the mandibles in the control and experimental groups. A vertical osteotomy was performed in the right mandible between the molars and the mandibular ramus using a fissure steel bar. Once bicortical holes were drilled in both fragments, the distractor was fixed with titanium bone screws (Figure 1). ${ }^{9,10}$

All surgical and distraction osteogenesis procedures were performed by the same researcher.

\section{Distraction protocol}

The distraction procedure was executed with a latency phase of 5 days after the surgical application, and the distraction was completed in 10 days. The distractor for each subject was activated twice a day during the distraction phase, with each turn resulting in a $0.175-\mathrm{mm}$ distraction every 12 hours. The animals were euthanized after the 4-week consolidation phase to maintain the regenerated bone tissue., ${ }^{2,4}$

\section{Histopathologic analysis}

For the remaining subjects, the original distracted bone area and the surrounding bone tissue were subjected to histomorphometric and immunohistochemical analyses. Briefly, specimens were fixed in $10 \%$ formaldehyde for $72 \mathrm{~h}$, demineralized in $10 \%$ formic acid, dehydrated, embedded in paraffin wax, and sectioned. The sections were used for haematoxylin and eosin and Masson trichrome staining and microscopic analysis. Starting from the centre of each specimen, serial sections were cut using a microtome, then stained. Sections that were $6-\mu \mathrm{m}$ thick, corresponding to the bone distraction area, were evaluated via light microscopy; osteoblasts and osteoclasts were scored numerically. ${ }^{11}$ In addition, new bone formation was determined as follows: the ratio (\%) of regenerated bone areas to all parts of the distracted area was calculated using an image program. ${ }^{12}$ All images obtained from the histological specimens were taken with a digital camera attached to a light microscope, and saved on a computer using the original magnification. The Olympus DP71 software imaging system was used for the histomorphometric analysis. All the histopathological procedures and analysis were done by the same pathologist.
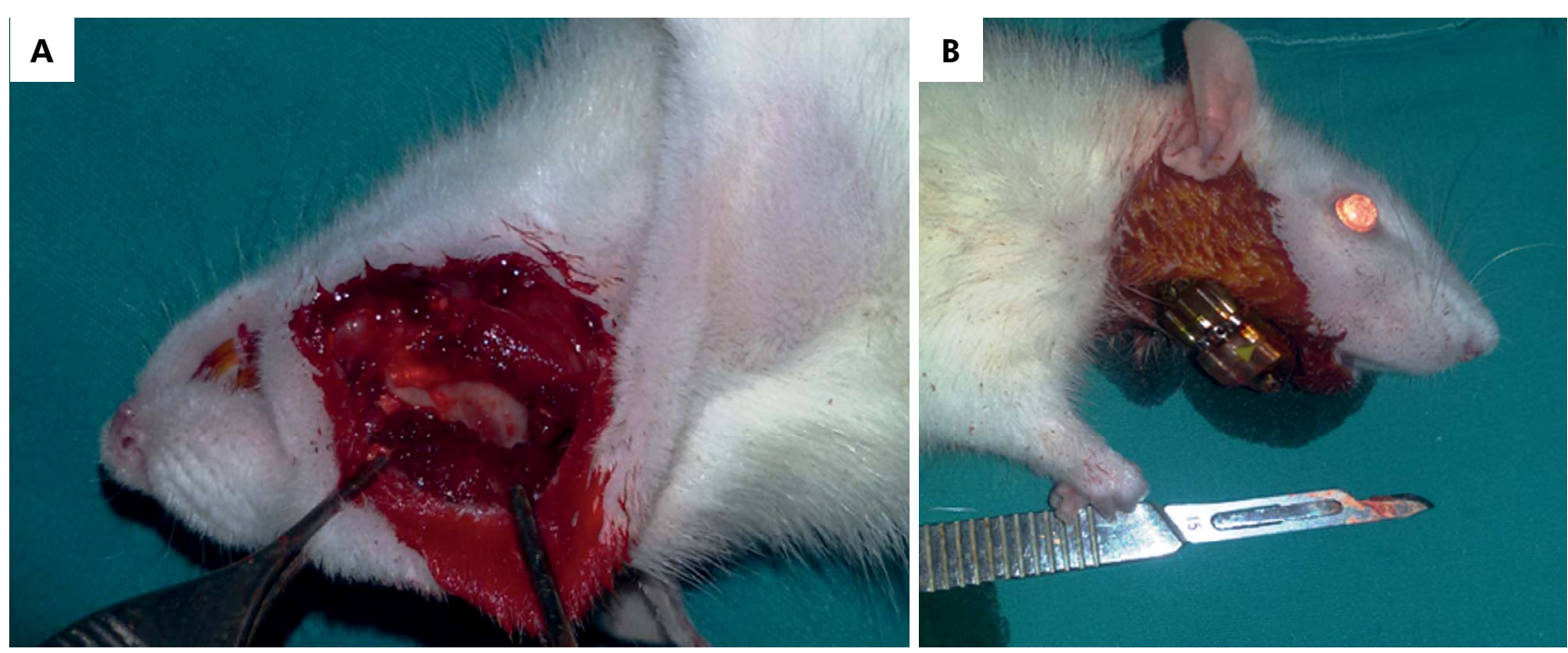

Figure 1. a) Surgical approach of the rat mandible. After the submandibular incision, the subcutaneous tissues and muscles were dissected and mandibular bone was reached. b) After the fixation of the distractor, the subcutaneous tissues and muscles were replaced in their original positions and the surgical area was washed with povidone iodine solution. 


\section{Immunohistochemical analysis}

After euthanasia, the specimens were fixed by perfusion, decalcified, and embedded in paraffin as described previously. The sections were incubated in an oven at $60^{\circ} \mathrm{C}$ for $10 \mathrm{~min}$, and $4-\mu \mathrm{m}$ longitudinal sections were cut. The sections were then placed in an automatic staining machine for OPN and VEGF immunohistochemical staining. Following the primary antibody procedure, sections were washed with water and stored in Ultramount mounting medium. Immunohistochemical staining was performed as follows: the staining ratio (\%) of OPN and VEGF in the regenerated bone areas was calculated with the image analysis program. ${ }^{13}$ All images obtained from the histological specimens were taken with a digital camera attached to a light microscope and saved on a computer using the original magnification. The Olympus DP71 software imaging system was used for the immunohistochemical analysis. All the immunohistochemical procedures and analyses were carried out by the same pathologist.

\section{Statistical analysis}

In the next stage of the experiment, SPSS software v.22 was used to conduct statistical analyses. Statistical differences between the groups were determined using the Kruskal-Wallis test. In binary comparisons, the Mann-Whitney $U$ test was used to determine the origin of the difference between the groups, and $\mathrm{p}$ $<0.05$ was considered indicative of a statistically significant difference.

\section{Results}

As noted previously, during the experimental protocol, three rats in the control group and four rats in each treatment group died. Thus, the study was completed with seven rats in the control group and six rats in each treatment group.

Among the specimens that were examined, the percentages of new bone formation (NBF) in the control (Figure 2a, 2b, 2c, 2d), MLT-D1 (Figure 2e, 2f, $2 \mathrm{~g}$, 2h), and MLT-D2 (Figure 2i, 2j, 2k, 2l) groups were $32.14 \%, 36.67 \%$, and $43.33 \%$, respectively. The increase in the percentage of NBF was significantly higher in the MLT-D1 and MLT-D2 groups than in the control group $(\mathrm{p}<0.05)$. Furthermore, the percentage of NBF was higher in the MLT-D2 group than in the MLTD1 group $(\mathrm{p}<0.05)$ (Table).

There were 21 osteoblasts in the control group (Figure 2a), 31.5 in the MLT-D1 group (Figure 2e), and 39.83 in the MLT-D2 group (Figure 2i). The increase in the number of osteoblasts in the MLT-D1 and MLTD2 groups was significantly higher than that in the control group $(p<0.05)$. Moreover, there were more osteoblasts in the MLT-D2 group than in the MLTD1 group $(\mathrm{p}<0.05)$ (Table).

Similarly, the osteoclast numbers were $0.43,2.67$, and 3.33 in the control group (Figure 2a), MLT-D1 group (Figure 2e), and MLT-D2 group (Figure 2i), respectively. The difference between the control and MLT-D1 and MLT-D2 groups was statistically significant $(\mathrm{p}<0.05)$. No statistically significant differences in the osteoclast numbers were seen between the MLT-D2 and MLTD1 groups $(\mathrm{p}>0.05)$ (Table).

Results of the immunohistochemical analysis revealed that the percentages of OPN expression were $28.57 \%, 35.83 \%$, and $43.33 \%$ in the control (Figure 2c), MLT-D1 (Figure 2g), and MLT-D2 groups (Figure 2k), respectively. The VEGF rates were $27.86 \%, 35 \%$, and $43.33 \%$ in the control (Figure 2d), MLT-D1 (Figure 2h), and MLT-D2 (Figure 2l) groups, respectively. For both immunohistochemical molecules, the difference between the control group and the MLT-D1 and MLT-D2 groups was statistically significant $(p<0.05)$. There were no significant differences between the MLT-D2 and MLT-D1 groups ( $p>0.05)$.

\section{Discussion}

Various studies have reported that melatonin stimulates the osteogenic activity of bone tissue., ${ }^{7,1,15,16,17}$ Nevertheless, the effects of systemic melatonin application in DO have rarely been studied. ${ }^{8}$ The histopathological and immunohistochemical results of this study reveal that melatonin could increase bone formation in mandibular DO in rats. In one in vitro study, Roth et al. ${ }^{17}$ have reported that melatonin can increase MC3T3-E1 cell differentiation and mineralization. Additionally, preliminary research has reported that melatonin can induce the differentiation 
of primary osteoblasts in vitro, verifying that the actions of melatonin are not limited to transformed cells. ${ }^{17}$ Furthermore, melatonin increased procollagen type I c-peptide expression in normal bone cells and could modulate the production of rat bone tissue sialoprotein in pre-osteoblast cell lines in
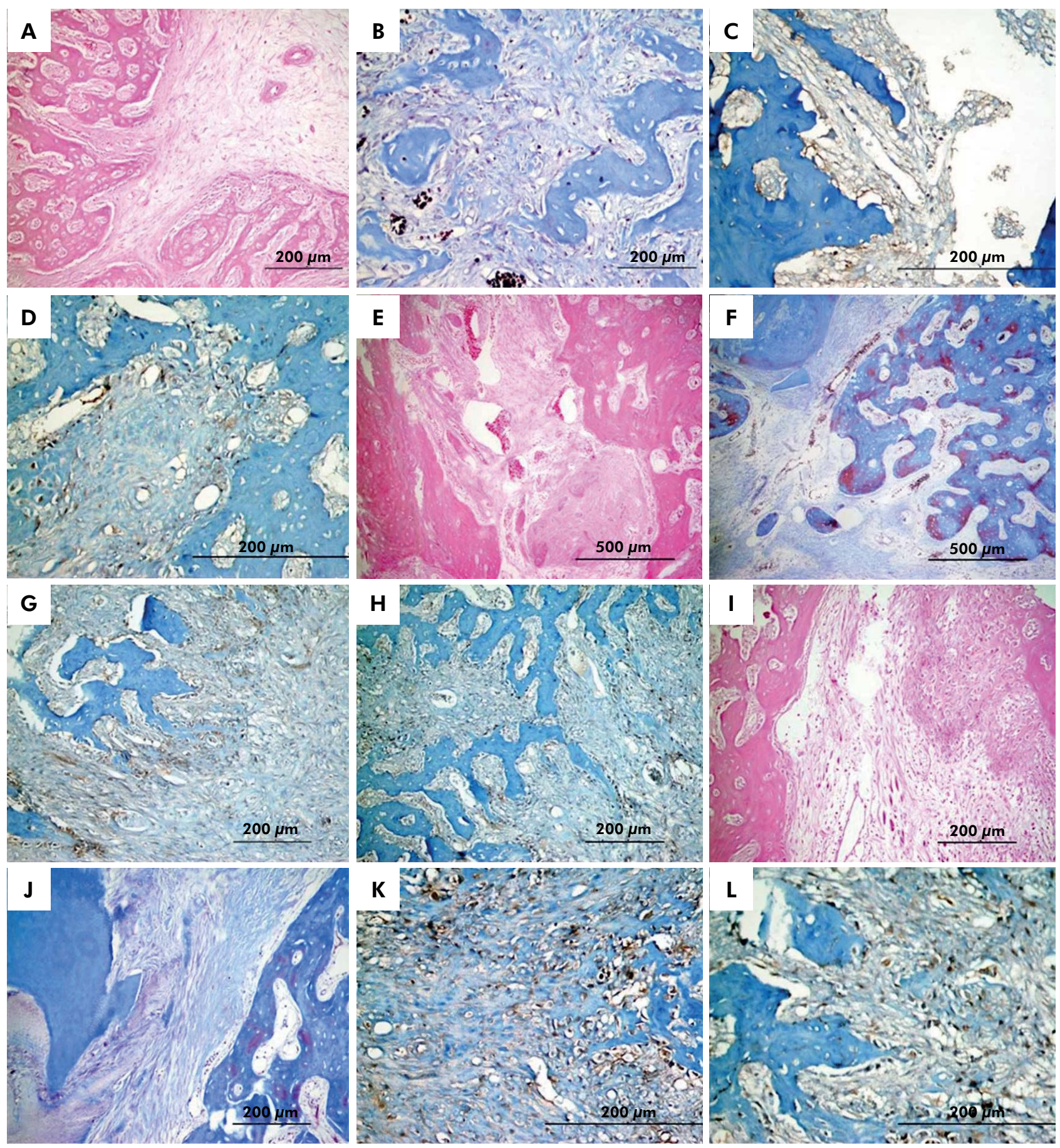

Figure 2. Histological and immunohistochemical $(\mathrm{IHC})$ evaluation of the distraction regeneration. In all the groups distraction areas-between the two bone fragments area-were filled with new bone tissues. Control group: (a) HE, haematoxylin and eosin; (b) MT, Masson trichome; (c) IHC-OPN, osteopontin; (d) IHC-VEGF, vascular endothelial growth factor. Melatonin dose 1 group: (e) HE, (f) MT, (g) IHC-OPN, (h) IHC-VEGF. Melatonin dose 2 group: (i) HE, (i) MT, (k) IHC-OPN, (l) IHC-VEGF. 
Table. Histomorphometric and immunohistochemical analysis of the groups.

\begin{tabular}{lccccc}
\hline Groups & NBF (\%) & OB (number) & OC (number) & OPN (\%) & VEGF (\%) \\
\hline MLT-D-2 $(n=6)$ & $43.33(40-50)^{a, b}$ & $39.83(35-48)^{a, b}$ & $3.33(2-6)^{a}$ & $43.33(40-50)^{a, b}$ & $43.33(40-50)^{a, b}$ \\
MLT-D-1 $(n=6)$ & $36.67(35-40)^{a}$ & $31.5(25-36)^{a}$ & $2.67(2-4)^{a}$ & $35(30-40)^{a}$ & $35.83(30-40)^{a, b}$ \\
Control $(n=7)$ & $32.14(30-35)$ & $21(17-25)$ & $0.43(0-2)$ & $28.57(25-35)$ & $27.86(25-35)$ \\
p-value $^{*}$ & 0.001 & 0.001 & 0.003 & 0.001 & 0.001 \\
\hline
\end{tabular}

("Kruskall Wallis), "Statistically significantlly difference compared with the Controls (Mann Whitney-U); bStatistically significantlly difference compared with the MLT-D-1 (Mann Whitney-U). p < 0.05: Statistically significant, Datas presented (mean) (min-max). MLT: Melatonin; D: Dose

vitro. Melatonin was also found to promote the gene expression of sialoprotein and other bone marker proteins, including alkaline phosphate, secreted protein, osteocalcin, and osteopontin. ${ }^{17}$ Nakade et al. ${ }^{18}$ showed that melatonin stimulates type 1 collagen synthesis and proliferation in human bone cells, and that it may even stimulate bone formation. Koyama et al. ${ }^{19}$ revealed that melatonin inhibits bone resorption and increases bone mass by decreasing the receptor activator of nuclear factor kappa-B ligand (RANKL)-mediated osteoclast formation. Therefore, melatonin appears to be capable of promoting osteoblast differentiation and matrix mineralization in vitro. ${ }^{19}$ The results of our research were in agreement with those of Koyama et al. ${ }^{19}$, who investigated the effects of systemic melatonin application on bone tissue in mice in vivo. They stated that pharmacological doses of melatonin increased bone tissue mass, bone tissue mineral density, and trabecular bone volume. The authors reported that these changes obviously stemmed from an osteoblastic effect of the melatonin hormone. Moreover, Satomura et al. ${ }^{16}$ demonstrated the systemic osteogenic effects of intraperitoneal melatonin administration in their experimental study. Specifically, they reported an increase in the volume of newly formed cortical bone tissues of mouse femora. Another experimental study reported that injecting young mice with 5 or $50 \mathrm{mg} / \mathrm{kg}$ of melatonin daily for four weeks significantly increased bone mineral density and the thickness and volume of trabeculae. ${ }^{19}$

Similar to the results of previous studies, we administrated melatonin systematically in two different concentrations and found that the osteogenic effects of melatonin could be explained by its inductive effect on osteoblastic cells. The osteoblastic properties of melatonin have been examined in several other studies, and our immunohistochemical
(OPN and VEGF) and histopathologic (NBF and $\mathrm{OB})$ findings were comparable to what has been reported previously. ${ }^{5,20,21}$ For instance, during bone tissue development and remodelling, OPN and VEGF participate in osteoblast maturation and bone matrix mineralization, with the osteoblastic differentiation and maturation being stimulated by VEGF. Moreover, OPN is expressed by osteoblast cells before matrix mineralization, and this molecule is present as one of the important non-collagenous elements of the extracellular matrix. OPN and VEGF also contribute to bone tissue reparation. ${ }^{22}$ Here, a weekly injection of 25 and $50 \mathrm{mg} / \mathrm{kg}$ melatonin in rats for four weeks significantly increased newly regenerated bone tissues in the distracted areas. Consequently, these findings provide conclusive evidence that melatonin treatment could lead to an increase in bone volume in vivo.

Likewise, Erdem et al. ${ }^{8}$ reported in their experimental rat femur DO study that melatonin, caffeic acid, and phenyl ethyl ester all increased the maturation of NBF. They suggested that these effects likely stemmed from reducing the bone tissue's resorptive abilities by inhibiting NF- $\kappa \beta$ and free oxygen radicals, as melatonin is known to be a significant free oxygen radical scavenger, and free oxygen radicals disrupt the bone tissue regeneration process. They used $25 \mathrm{mg} / \mathrm{kg}$ melatonin in that study. We used 25 and $50 \mathrm{mg} / \mathrm{kg}$ melatonin in the present study. We aimed to investigate whether higher doses would shorten the maturation period and increase NBF in the mandibular bone in rats. Maturation of the new bone is a major concern in DO. ${ }^{2}$

Previous studies have demonstrated that melatonin is an effective osteoblastic material when used locally. For instance, Dundar et al. ${ }^{5}$ carried out an experimental rabbit study and found that local application of two 
different doses of melatonin in implant sockets during surgery increased bone implant connection. Munoz et al. ${ }^{15}$ conducted a study in which $4 \mathrm{IU}$ of recombinant human growth hormone and $1.2 \mathrm{mg}$ of lyophilized powdered melatonin were applied to bilateral implant sockets of dog mandibles prior to implantation. They found that melatonin and growth hormone synergistically enhanced new peri-implant bone formation in the early stages of the tissue healing process. ${ }^{15}$ In a similar study, Tresguerres et al. ${ }^{6}$ stated that a $3 \mathrm{mg}$ local melatonin application during surgical implant integration might increase trabecular bone implant connection as well as trabecular bone area density. These results were similar to those of Cutando et al. ${ }^{7}$, who applied $1.2 \mathrm{mg}$ of lyophilized melatonin locally into the mandibular implant sockets of dogs before implant placement; they reported an increase in the osseointegration of the implant.

In clinical research, El-Gammal et al. ${ }^{21}$ described results comparable to those of previous experimental studies. Beyond the local application of melatonin in implant research, Takechi et al. ${ }^{20}$ used melatonin systematically with local fibroblast growth factor-2, producing similar results in their experimental research. Specifically, they reported that systemic melatonin application with local fibroblast growth factor-2 resulted in an increase in peri-implant bone tissue formation in rats. As the research outlined here indicates, osseointegration is directly connected with osteoblastic activity, so our results confirmed the osteoblastic activity of systemic melatonin application.

The authors of the present work investigated the effect of melatonin on angiogenesis in wound healing in a previous experimental animal study. ${ }^{6,23}$ In addition to its immunomodulatory effects, melatonin was found to have a favourable effect on monocyte, cytokine, and fibroblast proliferation, all of which activate angiogenesis. Bone tissue healing is a complex process that involves cell proliferation, angiogenesis, blood circulation, and matrix remodelling. Of these, angiogenesis is the most important process; it is organized by several growth factors, including VEGF. ${ }^{23}$ This is highlighted in an experimental rat study conducted by Soybir et al. ${ }^{24}$, in which melatonin application was found to increase the number of blood vessels in wounds. Additionally, RamérezFernández et al. ${ }^{23}$ reported that melatonin could enhance bone cell proliferation due to its accelerating angiogenetic effects. The results of our present study also suggest that the regeneration of new bone was dependent on the blood supply in the bone tissue. Lastly, Yamada et al. ${ }^{25}$ reported that angiogenesis is an important factor in osteogenesis; in particular, angiogenesis can increase bone tissue regeneration.

\section{Conclusion}

The morphometrical and immunohistochemical outcomes in our study revealed a melatonin-induced dose-dependent increase in new bone regeneration in the melatonin groups compared with the control group. Thus, within the limitations of this in vivo study, it can be concluded that systemic melatonin administration during DO may stimulate NBF. However, more studies are required to further investigate the increase and stabilization of bone formation in a DO procedure.

\section{Acknowledgements}

The authors wish to extend their gratitude to Gulmaksan, Esdent Dental Implant Corporation, Izmir, Turkey for providing the titanium screws.

\section{References}

\footnotetext{
1. Tüz HH, Dolanmaz D, Pampu AA, Kişnişci RS, Günhan $O$. Histomorphometric evaluation of delayed changes in masseter muscle after lengthening the rabbit mandible by distraction osteogenesis. Oral Dis. 2009 Mar;15(2):142-7. https://doi.org/10.1111/j.1601-0825.2008.01467.x
}

\footnotetext{
2. Dundar S, Artas G, Acikan I, Yaman F, Kirtay M, Ozupek MF et al. Comparison of the Effects of Local and Systemic Zoledronic Acid Application on Mandibular Distraction Osteogenesis. J Craniofac Surg. 2017 Oct;28(7):e621-5. https://doi.org/10.1097/SCS.0000000000003629
} 
3. Pampu AA, Dolanmaz D, Tüz HH, Avunduk MC, Kişnişci RS. Histomorphometric evaluation of the effects of zoledronic acid on mandibular distraction osteogenesis in rabbits. J Oral Maxillofac Surg. 2008 May;66(5):905-10. https://doi.org/10.1016/i.joms.2007.12.004

4. Kocyigit ID, Coskunses FM, Pala E, Tugcu F, Onder E, Mocan A. A comparison of the low-laser versus low intensity pulsed ultrasound on new bone formed through distraction osteogenesis. Photomed Lase Surg.2012 Aug; 30(8):483-43. https://doi.org/10.1089/pho.2012.3263

5. Dundar S, Yaman F, Saybak A, Ozupek MF, Toy VE, Gul $M$ et al. Evaluation of effects of topical melatonin application on osseointegration of dental implant: an experimental study. J Oral Implantol. 2016 Oct;42(5):386-9. https://doi.org/10.1563/aaid-joi-D-16-00048

6. Tresguerres IF, Celemente C, Blanco L, Khraisat A, Tamimi F, Tresguerres JA. Effects of local melatonin application on implant osseointegration. Clin Implant Dent Relat Res. 2012 Jun;14(3):3959. https://doi.org/10.1111/j.1708-8208.2010.00271.x

7. Cutando A, Gómez-Moreno G, Arana C, Muñoz F, Lopez-Peña $M$, Stephenson J et al. Melatonin stimulates osteointegration of dental implants. J Pineal Res. 2008 Sep;45(2):174-9. https://doi.org/10.1111/i.1600-079X.2008.00573.x

8. Erdem M, Gulabi D, Sen C, Sahin SA, Bozdag E. Effects of caffeic acid phenethyl ester and melatonin on distraction osteogenesis: an experimental study. Springerplus. 2014 Jan;3(1):8. https://doi.org/10.1186/2193-1801-3-8

9. Sakagami N, Kobayashi T, Nozawa-Inove K, Oda K, Kojima T, Maeda T et al. A histologic study of deformation of the mandibular condyle caused by distraction in a rat model. Oral Surg Oral Med Oral Pathol Oral Radiol. 2014 Sep;118(3):28494. https://doi.org/10.1016/i.0000.2014.05.003

10. Kang SY, Deshpande SS, Donneys A, Rodriguez JJ, Nelson NS, Felice PA et al. Parathyroid hormone reverses radiation induced hypovascularity in a murine model of distraction osteogenesis. Bone. 2013 Sep;56(1):9-15. https://doi.org/10.1016/i.bone.2013.04.022

11. Küçük D, Ay S, Kara MI, Avunduk MC, Gümus C. Comparison of local and systemic alendronate on distraction osteogenesis. Int J Oral Maxillofac Surg. 2011 Dec;40(12):1395-400. https://doi.org/10.1016/j.ijom.2011.08.004

12. Acar AH, Alan H, Özgür C, Vardi N, Asutay F, Güler Ç. Is more cortical bone decortication effective on guided bone augmentation? J Craniofac Surg. 2016 Oct;27(7):1879-83. https://doi.org/10.1097/SCS.0000000000002932

13. Raposo-Ferreira TM, Salvador RC, Terra EM, Ferreira JH, Vechetti-Junior IJ, Tinucci-Costa M et al. Evaluation of vascular endothelial growth factor gene and protein expression in canine metastatic mammary carcinomas. Microsc Res Tech. 2016 Nov;79(11):1097-104. https://doi.org/10.1002/jemt.22763

14. López-Martínez F, Olivares Ponce PN, Guerra Rodríguez M, Martínez Pedraza R. Melatonin: bone metabolism in oral cavity. Int J Dent. 2012;2012:628406. https://doi.org/10.1155/2012/628406
15. Muñoz F, López-Peña M, Miño N, Gómez-Moreno G, Guardia J, Cutando A. Topical application of melatonin and growth hormone accelerates bone healing around dental implants in dogs. Clin Implant Dent Relat Res. 2012 Apr;14(2):226-35. https://doi.org/10.1111/j.1708-8208.2009.00242.x

16. Satomura K, Tobiume S, Tokuyama R, Yamasaki Y, Kudoh K, Maeda $E$ et al. Melatonin at pharmacological doses enhances human osteoblastic differentiation in vitro and promotes mouse cortical bone formation in vivo. J Pineal Res. 2007 Apr;42(3):2319. https://doi.org/10.1111/j.1600-079X.2006.00410.x

17. Roth JA, Kim BG, Lin WL, Cho MI. Melatonin promotes osteoblast differentiation and bone formation. J Biol Chem. 1999 Jul;274(31):22041-7. https://doi.org/10.1074/jbc.274.31.22041

18. Nakade O, Koyama H, Ariji H, Yajima A, Kaku T. Melatonin stimulates proliferation and type I collagen synthesis in human bone cells in vitro. J Pineal Res. 1999 Sep;27(2):106-10. https://doi.org/10.1111/j.1600-079X.1999.tb00603.x

19. Koyama H, Nakade O, Takada Y, Kaku T, Lau KH. Melatonin at pharmacologic doses increases bone mass by suppressing resorption through down-regulation of the RANKL-mediated osteoclast formation and activation. J Bone Miner Res. 2002 Jul;17(7):1219-29. https://doi.org/10.1359/jbmr.2002.17.7.1219

20. Takechi M, Tatehara S, Satomura K, Fujisawa K, Nagayama M. Effect of FGF-2 and melatonin on implant bone healing: a histomorphometric study. J Mater Sci Mater Med. 2008 Aug;19(8):2949-52. https://doi.org/10.1007/s10856-008-3416-3

21. El-Gammal MY, Salem AS, Anees MM, Tawfik MA. Clinical and Radiographic Evaluation of Immediate Loaded Dental Implants With Local Application of Melatonin: A Preliminary Randomized Controlled Clinical Trial. J Oral Implantol. 2016 Apr;42(2):119-25. https://doi.org/10.1563/aaid-joi-D-14-00277

22. Ramchandani D, Weber GF. Interactions between osteopontin and vascular endothelial growth factor: implications for skeletal disorders. Bone. 2015 Dec;81:7-15. https://doi.org/10.1016/i.bone.2015.05.047

23. Ramírez-Fernández MP, Calvo-Guirado JL, de-Val JE, Delgado-Ruiz RA, Negri B, Pardo-Zamora G et al. Melatonin promotes angiogenesis during repair of bone defects: a radiological and histomorphometric study in rabbit tibiae. Clin Oral Investig. 2013 Jan;17(1):147-58. https://doi.org/10.1007/s00784-012-0684-6

24. Soybir G, Topuzlu C, Odabaş O, Dolay K, Bilir A, Köksoy $F$. The effects of melatonin on angiogenesis and wound healing. Surg Today. 2003;33(12):896-901. https://doi.org/10.1007/s00595-003-2621-3

25. Yamada Y, Tamura T, Hariu K, Asano Y, Sato S, Ito $K$. Angiogenesis in newly augmented bone observed in rabbit calvarium using a titanium cap. Clin Oral Implants Res. 2008 Oct;19(10):1003-9. https://doi.org/10.1111/j.1600-0501.2008.01554.x 